\title{
Bioelectronic Nose Using Odorant Binding Protein-Derived Peptide and Carbon Nanotube Field-Effect Transistor for the Assessment of Salmonella Contamination in Food
}

\author{
Manki Son, ${ }^{\dagger}$ Daesan Kim, ${ }^{\ddagger}$ Jinkyung Kang ${ }^{\S}$ Jong Hyun Lim, ${ }^{\S}$ Seung Hwan Lee, ${ }^{\S}$ Hwi Jin Ko, \\ Seunghun Hong, ${ }^{*, \perp}$ and Tai Hyun Park ${ }^{*,+}, \S, \|, \# \mathbb{\infty}$ \\ ${ }^{\dagger}$ Interdisciplinary Program for Bioengineering, Seoul National University, Seoul 151-742, Korea \\ ${ }^{\ddagger}$ Department of Biophysics and Chemical Biology, Seoul National University, Seoul 151-742, Korea \\ ${ }^{\S}$ School of Chemical and Biological Engineering, Seoul National University, Seoul 151-742, Korea \\ "Bio-MAX Institute, Seoul National University, Seoul 151-818, Korea \\ ${ }^{\perp}$ Department of Physics and Astronomy and Institute of Applied Physics, Seoul National University, Seoul 151-747, Korea \\ \#Advanced Institutes of Convergence Technology, Suwon, Gyeonggi-do 443-270, Korea
}

Supporting Information

\begin{abstract}
Salmonella infection is the one of the major causes of food borne illnesses including fever, abdominal pain, diarrhea, and nausea. Thus, early detection of Salmonella contamination is important for our healthy life. Conventional detection methods for the food contamination have limitations in sensitivity and rapidity; thus, the early detection has been difficult. Herein, we developed a bioelectronic nose using a carbon nanotube (CNT) field-effect transistor (FET) functionalized with Drosophila odorant binding protein (OBP)-derived peptide for easy and rapid detection of
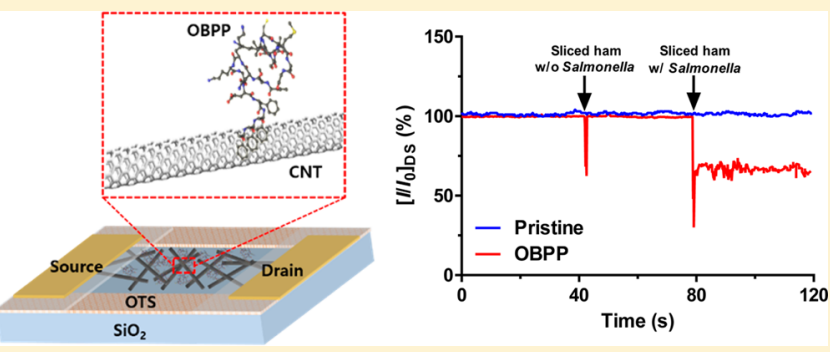
Salmonella contamination in ham. 3-Methyl-1-butanol is known as a specific volatile organic compound, generated from the ham contaminated with Salmonella. We designed and synthesized the peptide based on the sequence of the Drosophila OBP, LUSH, which specifically binds to alcohols. The C-terminus of the synthetic peptide was modified with three phenylalanine residues and directly immobilized onto CNT channels using the $\pi-\pi$ interaction. The p-type properties of FET were clearly maintained after the functionalization using the peptide. The biosensor detected $1 \mathrm{fM}$ of 3-methyl-1-butanol with high selectivity and successfully assessed Salmonella contamination in ham. These results indicate that the bioelectronic nose can be used for the rapid detection of Salmonella contamination in food.
\end{abstract}

Salmonella is a bacterium that causes food poisoning by the consumption of contaminated food. ${ }^{1}$ Symptoms include fever, malaise, abdominal pain, headache, myalgia, nausea, anorexia, and constipation, which threatens one's health. ${ }^{2}$ For the effective detection of Salmonella contamination, various methods such as enzyme immunoassay, ${ }^{3}$ antibody capture, ${ }^{4}$ DNA probes, ${ }^{5}$ and PCR assay ${ }^{6}$ have been developed. However, conventional methods have low sensitivity, thus limiting the early detection of pathogens. Gas chromatography-mass spectrometry (GC/MS) has also been used to analyze volatile organic compounds (VOCs) of food contaminated with Salmonella, because VOCs such as 3-methyl-1-butanol and 1hexanol were specifically detected in the headspace of Salmonella-contaminated ham. ${ }^{7}$ However, GC/MS still has disadvantages of long analytical time and complex operations with high cost.

Recently, bioelectronic sensors using olfactory receptors and nanomaterials were reported for the rapid and simple detection of chemicals. The biosensors mimic the natural smell sensing mechanism that selectively recognizes the odorants. The integration of biomolecules and nanomaterials, such as carbon nanotubes, ${ }^{8-15}$ conducting polymer, ${ }^{16,17}$ and graphene, ${ }^{18,19}$ could allow us to detect the minute change in the bioelectrical potentials. The biosensors, named as the bioelectronic nose, could be applied to disease diagnosis, ${ }^{12}$ environmental monitoring, ${ }^{15}$ and food safety control. ${ }^{10,11,13}$

Herein, we present another bioelectronic nose, which can be used for the detection of Salmonella contamination in ham, using Drosophila odorant binding protein (OBP)-derived peptide and carbon nanotube field-effect transistor (CNTFET). When odor molecules stimulate the olfactory system, OBP, which is a soluble protein in olfactory mucus, binds with the odorant and transfers it to olfactory receptors. ${ }^{20}$ Combinatorial binding patterns of olfactory receptors and

Received: August 22, 2016

Accepted: November 14, 2016

Published: November 14, 2016 
odorants send electrical signals to higher regions of the brain, and finally, smell is perceived. ${ }^{21}$ LUSH is an OBP of Drosophila that specifically binds to alcohols. ${ }^{22}$ The structure of LUSH was defined, and the specific binding site for alcohol was previously reported. ${ }^{23}$ OBPs have been used for the development of biosensors which were combined with surface plasmon resonance, ${ }^{24}$ quartz crystal microbalance, ${ }^{25}$ surface acoustic wave, ${ }^{26-28}$ cantilever, ${ }^{29}$ electrochemical impedance spectroscopy, ${ }^{30,31}$ and FET. ${ }^{32}$ In this study, the peptide, which is derived from LUSH, was synthesized by the modification of the Cterminus with three additional phenylalanine (Phe) residues, and it includes specific binding site residues of LUSH. The synthesized peptide was directly immobilized onto CNT surfaces via the $\pi-\pi$ stacking interactions between Phe residues and CNTs. ${ }^{33,34}$ The peptide-based bioelectronic nose that is inspired by the insect olfactory systems could detect 3-methyl1-butanol with high specificity and selectivity. Furthermore, the bioelectronic nose could be used for the rapid assessment of Salmonella contamination by simply diluting ham samples.

\section{EXPERIMENTAL SECTION}

The CNT-FET was fabricated via the photolithography method as previously reported. ${ }^{35}$ Briefly, AZ5214 photoresist (PR) was used to fabricate a pattern on a $\mathrm{SiO}_{2}$ wafer. The wafer was dipped into octacyltrichlorosilane (OTS) solution (OTS/ hexane $=1: 500)$ for $7 \mathrm{~min}$. The wafer was rinsed with hexane, acetone, and ethanol to remove the PR patterns. The OTSpatterned substrate was incubated in swCNT solution (0.02 $\mathrm{mg} / \mathrm{mL}$ in 1,2-dichlorobenzene) for $30 \mathrm{~s}$, and CNTs were specifically absorbed to the substrate. Afterward, $\mathrm{Pd} / \mathrm{Au}(10 / 30$ $\mathrm{nm}$ ) electrodes were fabricated and passivated using PR to protect them from contacting the solution. The Drosophila OBP-derived peptide (TKCVSLMAGTVNKKGEFFFF) was synthesized with purity higher than $90 \%$ by Peptron (Korea). The peptide was diluted at $1 \mu \mathrm{g} / \mathrm{mL}$ in distilled water and stored at $-20{ }^{\circ} \mathrm{C}$. For the immobilization of the peptide, $1.5 \mu \mathrm{L}$ of peptide solution was placed on the CNT channel and incubated for $4 \mathrm{~h}$ at room temperature. After the immobilization process, unbound peptides were washed out 2-3 times with distilled water. An atomic force microscopy (AFM) system (MFP-3D, Asylum Research, USA) was used to analyze the immobilization of the peptide. The pristine CNT channel was imaged at a scan rate of $0.2 \mathrm{~Hz}$. After the peptide immobilization, the CNT channel was imaged again at $0.05 \mathrm{~Hz}$.

All the odorants were sequentially diluted to 1:10 using distilled water and stored at $4{ }^{\circ} \mathrm{C}$ until used. Sliced ham (Korea) was purchased from a local market and stored at $4{ }^{\circ} \mathrm{C}$. To prepare a Salmonella-contaminated food sample, $4 \times 10^{7}$ CFU $\mathrm{mL}^{-1}$ of Salmonella typhimurium LT2 was spiked on sliced ham samples, and then, the ham samples were stored at $25{ }^{\circ} \mathrm{C}$ for 4 days. $^{36}$ After the storage, $1 \mathrm{~mL}$ of distilled water was added to $1 \mathrm{mg}$ of the food samples and diluted to 1:100 before use in the experiments. ${ }^{11}$ For measuring the current signal, 20 $\mu \mathrm{L}$ of distilled water was added on the CNT channel, and $2 \mu \mathrm{L}$ of odorant or food samples was injected into the CNT channels using micropipettes. A bias voltage of $0.1 \mathrm{~V}$ was applied to the source and drain electrodes, and the gate voltage was grounded. Current signals were monitored using a $2326 \mathrm{~A}$ source meter (Keithley, USA) and a MST 800 probe station (MS TECH, Korea). The bioelectronic sensors were used for only one measurement.

\section{RESULTS AND DISCUSSION}

For the detection of 3-methyl-1-butanol, which is an indicator of Salmonella contamination, Drosophila OBP-derived peptide (OBPP) has to be synthesized on the basis of the sequence and structure in the Drosophila OBP, LUSH. The three-dimensional structure of the LUSH was previously identified. ${ }^{23} \mathrm{Thr} 57$, Ser52, and Thr48 form a network of hydrogen bonds with alcohols providing a structural motif to increase binding affinity. Another research group also reported that Thr57 and Phe64 are essential amino acid residues that bind with ligands. ${ }^{25}$ The sequence of the synthesized peptide was TKCVSLMAGTVNKKGEFFFF. The three Phe residues were added to the Cterminus of the peptide for the immobilization onto CNTs via the $\pi-\pi$ stacking interaction. Only the original part of the peptide is free, while the three Phe residues are immobilized on CNT. Therefore, it is considered that the Phe addition does not change the three-dimensional structure and the binding affinity of the peptide. Moreover, the peptide isoelectric point calculated with Compute $\mathrm{pI} / \mathrm{Mw}^{37}$ was not changed and was maintained at 9.19 even after the addition of three Phe residues, since Phe is an uncharged residue. The peptide was directly immobilized on the CNT channel as a self-assembled monolayer, so that the field-effect can occur when the structure of the peptide is changed with the binding of 3-methyl-1butanol (Figure 1). The AFM images of the CNT channel were

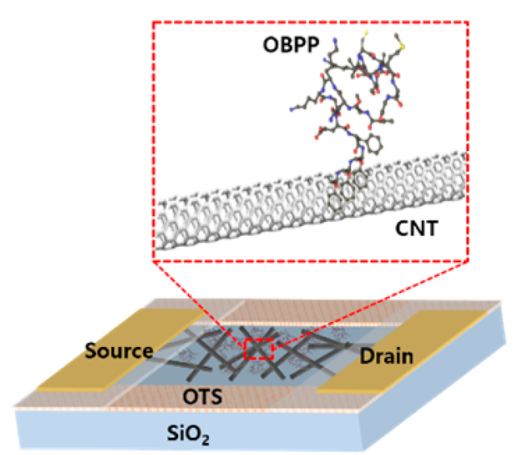

Figure 1. Schematic diagram of the bioelectronic nose using carbon nanotube field-effect transistor functionalized with odorant binding protein-derived peptide. The peptides were directly immobilized via $\pi-\pi$ interactions between Phe residues and CNTs.

measured before and after the immobilization of the peptide (Figure 2A). The height of the CNTs was scanned along the 5 $\mu \mathrm{m}$ region, and the average value of the height difference between two cases was calculated. The average height along the $5 \mu \mathrm{m}$ region increased by $2.05 \pm 2.01 \mathrm{~nm}$ ( $p$-value 0.0079$)$ (Figure 2B) after the immobilization process. Because the peptide length calculated with Hydropathy plot $^{38}$ was $2.19 \mathrm{~nm}$, it is considered that the peptides were appropriately immobilized on the CNT channel.

To analyze the electrical characteristic of the biosensor, the drain-source current-voltage $\left(I_{\mathrm{DS}}-V_{\mathrm{DS}}\right)$ curves of the CNT channel were measured before and after the immobilization of the peptide (Figure 3A). The resistance of the CNT channel was increased from $2.11 \times 10^{6}$ to $3.98 \times 10^{6} \Omega$, and the linearity of the $I_{\mathrm{DS}}-V_{\mathrm{DS}}$ curve was maintained. When we increased the liquid gate voltage in distilled water, the p-type FET characteristic was observed even after the immobilization process showing the decrease in the current $\left(I_{\mathrm{DS}}\right)$ with increasing gate voltage $\left(V_{\mathrm{G}}\right)$ (Figure $3 \mathrm{~B}$ ). These results indicate that the peptide was successfully immobilized onto the CNT 
(A)

\section{Pristine}

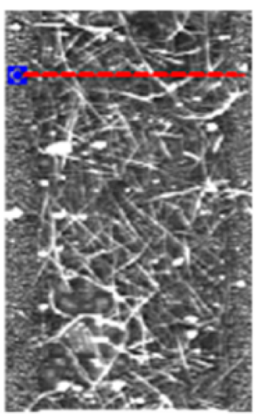

\section{OBPP}

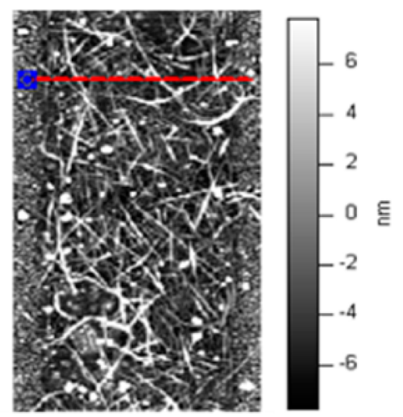

(B)

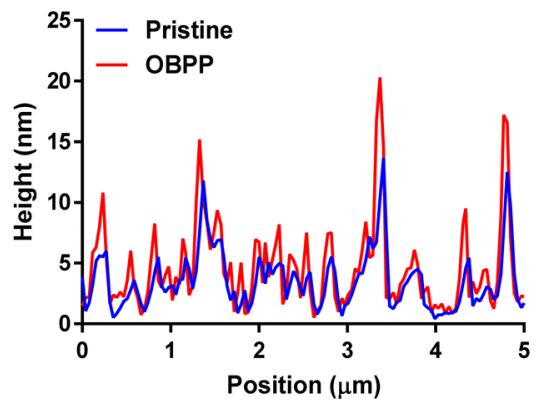

Figure 2. Fabrication of the peptide-based CNT-FET. (A) AFM images and (B) height profiles of CNT channels before and after the immobilization of OBPP.

(A)

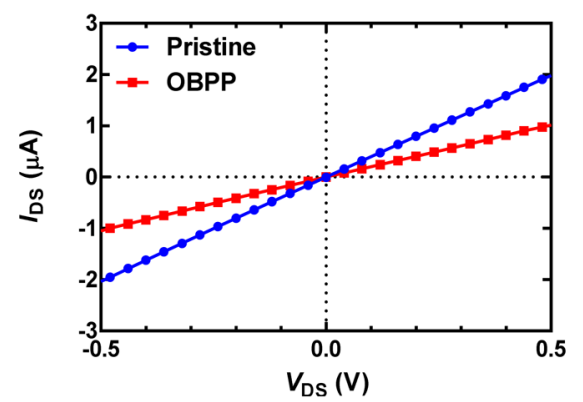

(B)

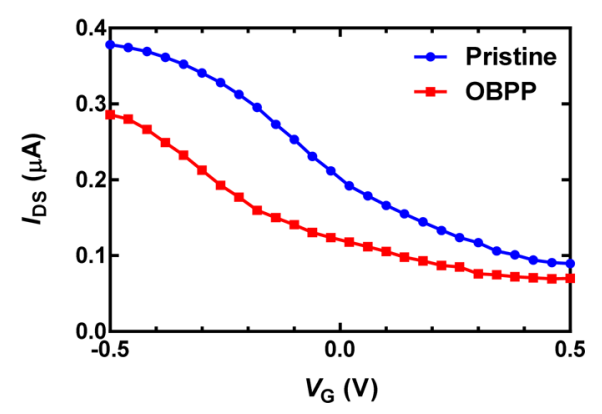

Figure 3. Electrical properties of the peptide-based CNT-FET. (A) Current-voltage curves and (B) liquid gate profiles of the CNT channels before and after the immobilization of OBPP. channel and the biosensor could be used for the detection of odorants.

The sensitivity and selectivity of the biosensor was demonstrated using 3-methyl-1-butanol, a standard odor that indicates Salmonella contamination in ham. Twenty $\mu \mathrm{L}$ of distilled water was placed on the CNT channel of the biosensor, and different concentrations of the odor solution were sequentially injected. Figure 4A shows the real-time

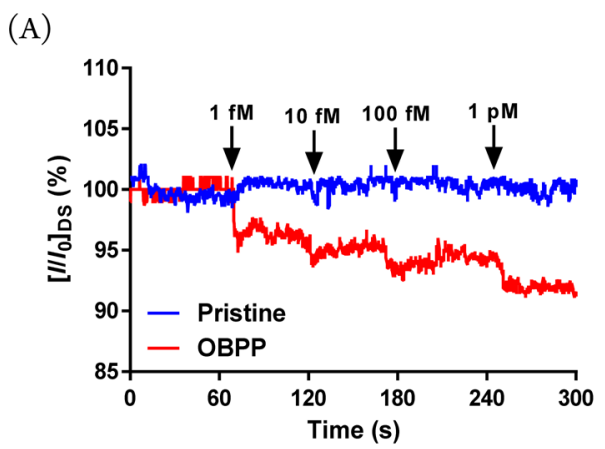

(B)

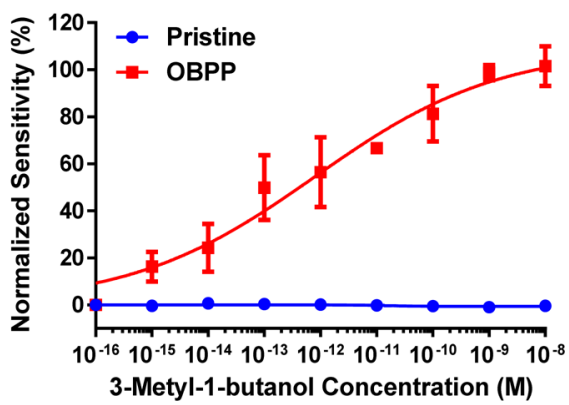

Figure 4. (A) Real-time conductance changes to various concentrations of 3-methyl-1-butanol using the pristine and OBPP-functionalized CNT-FET. (B) Dose-response curves of the bioelectronic nose to 3-methyl-1-butanol. The signals were normalized by the maximum response of the biosensor $\left(10^{-8} \mathrm{M}\right)$. Error bars, s.e.m. of three replicates.

conductance change with various concentrations of 3-methyl-1butanol. The signal intensity is the difference between the final current value and the initial baseline, which is the initial current value at $0 \mathrm{M}$ of 3-methyl-1-butanol. Prior to the injection of the distilled water, the diluent did not show significant change (data not shown), and no signal changes were observed at lower concentration than $1 \mathrm{fM}$. The biosensor exhibited the detection limit of $1 \mathrm{fM}$, and the signal increased with additional injection of higher concentrations. No significant signals were observed for the pristine CNT. The dose-dependent response of the biosensor was normalized by its maximum value and analyzed using the Langmuir isotherm model as previously reported (Figure 4B). ${ }^{11}$ The calculated equilibrium constant, $K_{\mathrm{d}}$, was $5.25 \times 10^{13} \mathrm{M}^{-1}$. Previously, we have tested the sensor selectivity with different lengths of carbon chains. ${ }^{8,16}$ Herein, we focused on the functional group selectivity of the sensor, since LUSH is the OBP that specifically binds with alcohol, which has a hydroxyl group. Six control compounds, with chemical structures similar to 3-methyl-1-butanol, did not show any response for both the pristine CNT and the OBPPfunctionalized CNT (Figure 5A). LUSH is the OBP that specifically binds to alcohol. OBPP is the synthetic peptide 
(A)

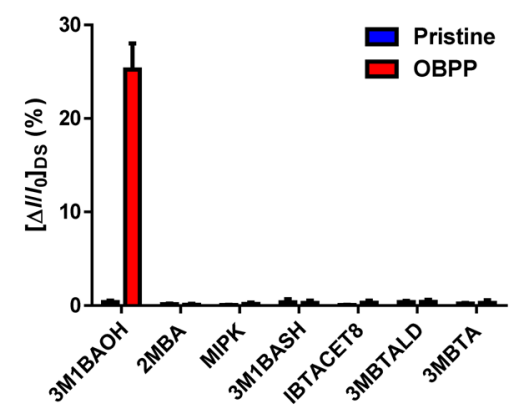

(B)

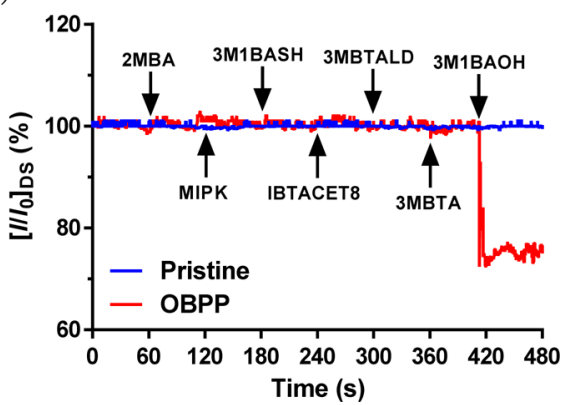

Figure 5. (A) Specific recognition of 3-methyl-1-butanol from other molecules in real-time. Five odorants including 3-methyl-1-butanol were applied to the bioelectronic nose in real-time for testing the selectivity of the biosensor, and all odorant concentrations were fixed at $1 \mathrm{nM}$. (B) Quantitative comparison of conductance changes with the injection of odor molecules. Error bars, s.e.m. of three replicates. The abbreviations used are as follows: $3 \mathrm{M} 1 \mathrm{BAOH}$, 3-methyl-1butanol; 2MBA, 2-methylbutane; MIPK, methyl isopropyl ketone; 3M1BASH, 3-methyl-1-butanethiol; IBTACET8, isobutyl acetate; 3MBTALD, 3-methylbutanal; 3MBTA, 3-methylbutanoic acid.

derived from the LUSH. The OBPP has the specific alcoholbinding site of the $\mathrm{LUSH}^{23}$ so that the bioelectronic nose can selectively distinguish relevant odorants. Quantitative comparison of 3-methyl-1-butanol to the control compounds shows that the biosensor selectively responded to 3-methyl-1-butanol (Figure 5B). These results indicate that the biosensor could sensitively and selectively detect the specific odorant generated from the Salmonella-contaminated ham.

When ham is contaminated by Salmonella, 3-methyl-1butanol is generated and this molecule is regarded as an indicator of contamination. ${ }^{7}$ To verify that the biosensor can be used for the assessment of food contamination, fresh and Salmonella-contaminated ham samples were injected to the biosensor. While the biosensor did not respond to a fresh ham sample, a significant signal was observed with a response time of less than $2 \mathrm{~s}$ when a Salmonella-contaminated ham sample was injected (Figure 6A). The pristine CNT did not respond to the Salmonella-contaminated ham sample as expected. Furthermore, inactivated Salmonella did not show any responses to the sensor (Figure S1). Thus, the detection of Salmonella-contaminated ham samples was available with the OBPP-functionalized CNT channel (Figure 6B). The simple manipulation and rapid detection of the biosensor allowed for the effective assessment of ham contamination by Salmonella.

\section{CONCLUSION}

In this study, an OBPP-based CNT biosensor was developed for the rapid assessment of Salmonella contamination in ham.
(A)

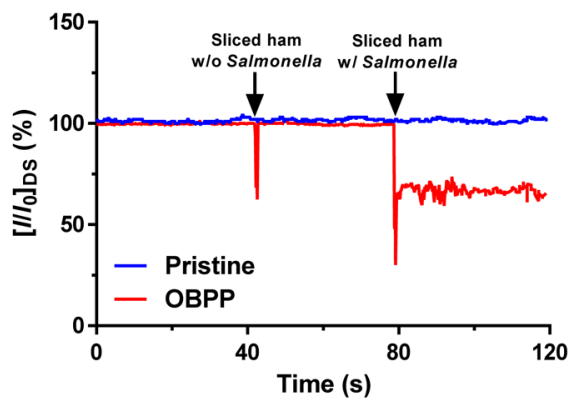

(B)

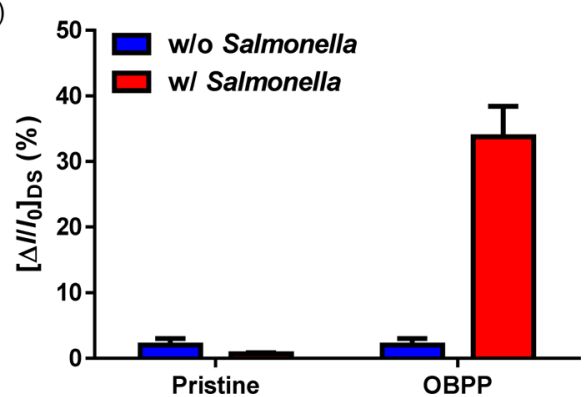

Figure 6. (A) Real-time detection of Salmonella contamination in sliced ham. (B) Quantitative comparison of conductance changes with the injection of noncontaminated and contaminated ham samples. Error bars, s.e.m. of three replicates.

LUSH is known as a Drosophila odorant binding protein to bind with alcohols. LUSH (Drosophila OBP)-derived peptide, containing essential amino acid residues for binding to alcohols, was synthesized with three additional phenylalanine residues at the C-terminus of the peptide and directly immobilized onto CNT channel of the biosensor via $\pi-\pi$ interactions between Phe residues and CNTs. The peptide-based biosensor sensitively detected 3-methyl-1-butanol at a concentration of $1 \mathrm{fM}$ and selectively distinguished the target odor molecule from other compounds with similar structures. Moreover, the biosensor detected the Salmonella-contaminated ham sample without a complex pretreatment process. Taken together, the bioelectronic nose, which relies on the peptide based on the insect OBP, can be used for the detection of Salmonella contamination in ham with a rapid and simple process. It is also expected that this approach can be applied to various food samples by using other OBPs or olfactory receptors which specifically bind with different food contaminators.

\section{ASSOCIATED CONTENT}

\section{S Supporting Information}

The Supporting Information is available free of charge on the ACS Publications website at DOI: 10.1021/acs.analchem.6b03284.

Real-time response of pristine and OBPP-functionalized CNT-FET to inactivated Salmonella (Figure S1) (PDF)

\section{AUTHOR INFORMATION}

\section{Corresponding Authors}

*E-mail: seunghun@snu.ac.kr.

*E-mail: thpark@snu.ac.kr. 


\section{ORCID ${ }^{\oplus}$}

Tai Hyun Park: 0000-0003-4254-0657

\section{Author Contributions}

The manuscript was written through contributions of all authors. All authors have given approval to the final version of the manuscript.

\section{Notes}

The authors declare no competing financial interest.

\section{ACKNOWLEDGMENTS}

This research was supported by the Public Welfare \& Safety Research Program through the National Research Foundation of Korea (NRF) funded by the Ministry of Science, ICT \& Future Planning (2014039771), the National Research Foundation of Korea (NRF) grant funded by the Korea government (MSIP) (2014053108, 2013M3A6B2078961), and the Center for Integrated Smart Sensors funded by the Ministry of Science, ICT \& Future Planning as Global Frontier Project (CISS-2011-0031870).

\section{REFERENCES}

(1) Helms, M.; Vastrup, P.; Gerner-Smidt, P.; Molbak, K. BMJ. 2003, 326, 357-361.

(2) Coburn, B.; Grassl, G. A.; Finlay, B. B. Immunol. Cell Biol. 2007, $85,112-118$

(3) Robison, B. J.; Pretzman, C. I.; Mattingly, J. A. Appl. Environ. Microbiol. 1983, 45, 1816-1821.

(4) Ng, S. P.; Tsui, C. O.; Roberts, D.; Chau, P. Y.; Ng, M. H. Appl. Environ. Microbiol. 1996, 62, 2294-2302.

(5) Knight, I. T.; Shults, S.; Kaspar, C. W.; Colwell, R. R. Appl. Environ. Microbiol. 1990, 56, 1059-1066.

(6) Wang, L.; Shi, L.; Alam, M. J.; Geng, Y.; Li, L. Food Res. Int. 2008, $41,69-74$

(7) Luna, G.; Aparicio, R.; Garcia-Gonzalez, D. L. Food Chem. 2006, 97, 621-630.

(8) Kim, T. H.; Lee, S. H.; Lee, J.; Song, H. S.; Oh, E. H.; Park, T. H.; Hong, S. Adv. Mater. 2009, 21, 91-94.

(9) Jin, H. J.; Lee, S. H.; Kim, T. H.; Park, J.; Song, H. S.; Park, T. H.; Hong, S. Biosens. Bioelectron. 2012, 35, 335-341.

(10) Park, J.; Lim, J. H.; Jin, H. J.; Namgung, S.; Lee, S. H.; Park, T. H.; Hong, S. Analyst 2012, 137, 3249-3254.

(11) Lim, J. H.; Park, J.; Ahn, J. H.; Jin, H. J.; Hong, S.; Park, T. H. Biosens. Bioelectron. 2013, 39, 244-249.

(12) Lim, J. H.; Park, J.; Oh, E. H.; Ko, H. J.; Hong, S.; Park, T. H. Adv. Healthcare Mater. 2014, 3, 360-366.

(13) Ahn, J. H.; Lim, J. H.; Park, J.; Oh, E. H.; Son, M.; Hong, S.; Park, T. H. Sens. Actuators, B 2015, 210, 9-16.

(14) Lee, S. H.; Lim, J. H.; Park, J.; Hong, S.; Park, T. H. Biosens. Bioelectron. 2015, 71, 179-185.

(15) Son, M.; Cho, D. G.; Lim, J. H.; Park, J.; Hong, S.; Ko, H. J.; Park, T. H. Biosens. Bioelectron. 2015, 74, 199-206.

(16) Yoon, H.; Lee, S. H.; Kwon, O. S.; Song, H. S.; Oh, E. H.; Park, T. H.; Jang, J. Angew. Chem., Int. Ed. 2009, 48, 2755-2758.

(17) Lee, S. H.; Kwon, O. S.; Song, H. S.; Park, S. J.; Sung, J. H.; Jang, J.; Park, T. H. Biomaterials 2012, 33, 1722-1729.

(18) Kwon, O. S.; Song, H. S.; Park, S. J.; Lee, S. H.; An, J. H.; Park, J. W.; Yang, H.; Yoon, H.; Bae, J.; Park, T. H.; Jang, J. Nano Lett. 2015, 15, 6559-6567.

(19) Park, S. J.; Kwon, O. S.; Lee, S. H.; Song, H. S.; Park, T. H.; Jang, J. Nano Lett. 2012, 12, 5082-5090.

(20) Ihara, S.; Yoshikawa, K.; Touhara, K. Neuroscience 2013, 254, $45-60$.

(21) Firestein, S. Sci. Signaling 2004, 2004, pe15.

(22) Kim, M. S.; Repp, A.; Smith, D. P. Genetics 1998, 150, 711-721.

(23) Kruse, S. W.; Zhao, R.; Smith, D. P.; Jones, D. N. Nat. Struct. Biol. 2003, 10, 694-700.
(24) Vidic, J.; Grosclaude, J.; Monnerie, R.; Persuy, M. A.; Badonnel, K.; Baly, C.; Caillol, M.; Briand, L.; Salesse, R.; Pajot-Augy, E. Lab Chip 2008, 8, 678-688.

(25) Sankaran, S.; Panigrahi, S.; Mallik, S. Biosens. Bioelectron. 2011, 26, 3103-3109.

(26) Di Pietrantonio, F.; Cannata, D.; Benetti, M.; Verona, E.; Varriale, A.; Staiano, M.; D'Auria, S. Biosens. Bioelectron. 2013, 41, 328-334.

(27) Di Pietrantonio, F.; Benetti, M.; Dinca, V.; Cannata, D.; Verona, E.; D’Auria, S.; Dinescu, M. Appl. Surf. Sci. 2014, 302, 250-255.

(28) Di Pietrantonio, F.; Benetti, M.; Cannata, D.; Verona, E.; PallaPapavlu, A.; Fernandez-Pradas, J. M.; Serra, P.; Staiano, M.; Varriale, A.; D'Auria, S. Biosens. Bioelectron. 2015, 67, 516-523.

(29) Manai, R.; Scorsone, E.; Rousseau, L.; Ghassemi, F.; Possas Abreu, M.; Lissorgues, G.; Tremillon, N.; Ginisty, H.; Arnault, J. C.; Tuccori, E.; Bernabei, M.; Cali, K.; Persaud, K. C.; Bergonzo, P. Biosens. Bioelectron. 2014, 60, 311-317.

(30) Lu, Y.; Yao, Y.; Zhang, Q.; Zhang, D.; Zhuang, S.; Li, H.; Liu, Q. Biosens. Bioelectron. 2015, 67, 662-669.

(31) Lu, Y.; Li, H.; Zhuang, S.; Zhang, D.; Zhang, Q.; Zhou, J.; Dong, S.; Liu, Q.; Wang, P. Sens. Actuators, B 2014, 193, 420-427.

(32) Larisika, M.; Kotlowski, C.; Steininger, C.; Mastrogiacomo, R.; Pelosi, P.; Schutz, S.; Peteu, S. F.; Kleber, C.; Reiner-Rozman, C.; Nowak, C.; Knoll, W. Angew. Chem., Int. Ed. 2015, 54, 13245-13248.

(33) Chen, R. J.; Zhang, Y.; Wang, D.; Dai, H. J. Am. Chem. Soc. 2001, 123, 3838-3839.

(34) Poenitzsch, V. Z.; Winters, D. C.; Xie, H.; Dieckmann, G. R.; Dalton, A. B.; Musselman, I. H. J. Am. Chem. Soc. 2007, 129, 1472414732.

(35) Lee, M.; Im, J.; Lee, B. Y.; Myung, S.; Kang, J.; Huang, L.; Kwon, Y. K.; Hong, S. Nat. Nanotechnol. 2006, 1, 66-71.

(36) Kim, S.; Kim, M.; Ryu, S. Anal. Chem. 2014, 86, 5858-5864.

(37) Bjellqvist, B.; Hughes, G. J.; Pasquali, C.; Paquet, N.; Ravier, F.; Sanchez, J. C.; Frutiger, S.; Hochstrasser, D. Electrophoresis 1993, 14, $1023-1031$.

(38) Kyte, J.; Doolittle, R. F. J. Mol. Biol. 1982, 157, 105-132. 